\title{
1 Mosquito odorant receptor sensitive to natural spatial repellents and
}

$7 \quad{ }^{1}$ Present address: R\&D Department, Jinju Bioindustry Foundation, Jinju, South Korea

$8 \quad *$ Correspondence: wsleal@ucdavis.edu

9 Lead Contact: Walter S. Leal, wsleal@ucdavis.edu, phone: 530-752-7755 


\section{Abstract}

21 Previously, we have identified an odorant receptor (OR) from the southern house mosquito Culex

22 quinquefasciatus, CquiOR32, which responded to both odorants (agonists) and inhibitory

23 compounds (antagonists). CquiOR32/CquiOrco-expressing oocytes responded to methyl

24 salicylate and other odorants with inward (regular) currents but gave currents in the reverse

25 direction when challenged with eucalyptol and other inhibitors. To determine whether hitherto

26 unknown ORs show this intrareceptor inhibition, we have now examined two other receptors in

27 the same cluster, CquiOR27 and CquiOR28. We cloned and tested four variants of CquiOR28,

28 but none of the 250 compounds in our panel of odorants, including an Orco ligand candidate

29 (OCL12), elicited inward or outward currents. By contrast, CquiOR27/CquiOrco-expressing

30 oocytes gave robust, dose-dependent inward currents when challenged with $\gamma$-octalactone and

31 other odorants. On the other hand, octylamine and other phenolic compounds elicited dose-

32 dependent currents in the reverse direction. When stimulatory and inhibitory compounds were

33 presented in binary mixtures, $\gamma$-octalactone-elicited inward currents were attenuated in a dose-

34 dependent manner according to the concentration of octylamine. As part of our chemical ecology

35 approach, we tested the repellency activity of the best ligands in the surface landing and feeding

36 assay and a newly reported hand-in cage assay. Protection elicited by $\gamma$-octalactone did not differ

37 significantly from that of DEET at the same dose. In the hand-in cage assay, a cream formulation

38 of $\gamma$-octalactone showed $97.0 \pm 1.3 \%$ protection, with $47.6 \pm 8.3 \%$ and $1.4 \pm 0.7 \%$ landings per

39 trial in the hands covered with a control and $\gamma$-octalactone cream, respectively $(\mathrm{N}=8, \mathrm{p}=0.0078$,

40 Wilcoxon matched-pairs signed-rank test). 
Keywords

48 Culex quinquefasciatus, CquiOR27, CquiOR32, $\gamma$-octalactone, $\gamma$-decalactone, $\gamma$-dodecalactone

\section{1. Introduction}

51 Integration of chemical signals at the insect's peripheral olfactory system is one of the least

52 understood mechanisms of insect olfaction. There is growing evidence in the literature that in

53 moths (Kaissling, 2014), beetles (Nikonov and Leal, 2002), vinegar fly (Su et al., 2012),

54 mosquitoes (Tauxe et al., 2013), and other insect species, the firing of one odorant receptor

55 neuron (ORN; also referred to as olfactory sensory neuron, OSN), interferes with the signaling

56 of other ORNs. There is convincing evidence that in the vinegar fly, lateral inhibition of

57 collocated ORNs may be mediated by ephaptic coupling (Su et al., 2012). Still, other

58 mechanisms seem to play a role in signal integration. For example, inhibition may occur within

59 the same neuron with the same compound eliciting excitatory and inhibitory responses (de Brito

60 Sanchez and Kaissling, 2005). Recently, we discovered an odorant receptor from the Southern

61 house mosquito, Culex quinquefasciatus, CquiOR32, which responded to some compounds

62 with regular inward currents, whereas other compounds elicited currents in the reverse direction

63 (outward currents) (Xu et al., 2019). Specifically, CquiOR32/CquiOrco-expressing oocytes

64 responded in a dose-dependent manner to methyl salicylate and other odorants with inward

65 currents, whereas inhibitory compounds elicited currents in the reverse direction (outward

66 currents). When CquiOR32/CquiOrco-expressing oocytes were challenged with mixtures of

67 stimulatory and inhibitory compounds, inward currents were attenuated in a dose-dependent 
68 fashion. The higher the inhibitor dose, the smaller the inward currents; binary mixtures with

69 high concentrations of inhibitors elicited currents in the reverse direction. The data thus suggest

70 that intrareceptor inhibition occurs, although it remains to be determined whether odorant

71 (stimulant) and inhibitor bind to the same or different binding sites in CquiOR32 (Xu et al.,

72 2019). Additionally, we identified an orthologue of CquiOR32 in the genome of the yellow

73 fever mosquito, Aedes aegypti, AaegOR71, which showed intrareceptor inhibition, although no

74 inhibitor-elicited outward currents could be recorded (Xu et al., 2019).

75 Phylogenetic analysis suggests that three other $C x$. quinquefasciatus ORs belong to a

76 CquiOR32 cluster: CquiOR36, CquiOR27, and CquiOR28 (Taparia et al., 2017). Previously,

77 we have deorphanized CquiOR36, which is narrowly tuned to acetaldehyde and showed no

78 evidence of intrareceptor inhibition (Choo et al., 2018). We have now cloned CquiOR27 and

79 CquiOR28 and attempted to deorphanize these receptors using the Xenopus oocyte recording

80 system. CquiOR28/CquiOrco-expressing oocytes did not respond to any of the 250 compounds

81 in our panel of odorants, including the Orco ligand candidate OCL12 (Chen and Luetje, 2012)

82 (also known as VUAA3 (Taylor et al., 2012)). On the other hand, we recorded robust inward

83 currents from CquiOR27/CquiOrco-expressing oocytes challenged with $\gamma$-octalactone and other

84 odorants. By contrast, octylamine and other phenolic compounds elicited currents in the reverse

85 direction and, when presented as binary mixtures, attenuated the responses to $\gamma$-octalactone in a

86 dose-dependent manner. As part of our reverse chemical ecology approach (Leal, 2005), we

87 measured the behavioral responses of $C x$. quinquefasciatus to $\gamma$-octalactone in repellency

88 assays. Protection data obtained with the surface landing and feeding assay (Leal et al., 2017; 
$89 \mathrm{Xu}$ et al., 2014) and a newly reported hand-in cage assay suggest that $\gamma$-octalactone is a potent

90 mosquito repellent.

91

\section{2. Material and methods}

93 2.1. Insect preparations

94 Cx. quinquefasciatus used in this study were from a laboratory colony started in the 1950s

95 from adult mosquitoes collected in Merced, CA. This colony has been maintained at the

96 Kearney Agricultural Research Center, University of California, Parlier, CA, and kept for

97 almost ten years in Davis. Mosquitoes were maintained at $27 \pm 1^{\circ} \mathrm{C}, 75 \pm 5 \%$ relative humidity,

98 and under a photoperiod of 12:12 h.

99

100 2.2. RNA and DNA extraction, DNA synthesis, and cloning

101 Total RNA was extracted from one thousand 4-7 day-old female Cx. quinquefasciatus antennae

102 with TRIzol reagent (Invitrogen, Carlsbad, CA). Antennal cDNA was synthesized from $1 \mu \mathrm{g}$ of

103 antennal total RNA using SMARTerTM RACE cDNA amplification kit according to

104 manufacturer's instructions (Clontech, Mountain View, CA). To obtain full-length coding

105 sequences, PCRs were performed using the following gene-specific primers:

106 CqOR27-Fw: 5'- ATGGACACAGTCCGGTGGGCTTCA-3’;

107 CqOR27-Rv: 5'- TTACAACTTGTTAAGCAAATCTTTGAGAACCAAATAGTACGAG -3’

108 CqOR28-Fw: 5'- ATGGACGCCACCCAGCGAATCAAA-3';

109 CqOR28-Rv: 5'- TTACAGTTTGCTCAAAACGTCATTCAACACCAAATA-3'. 
110 PCR products were purified by a QIAquick gel extraction kit (Qiagen, Germantown, MD) and

111 then cloned into pGEM-T vector (Promega, Madison, WI). Plasmids were extracted by a

112 QIAprep spin miniprep kit (Qiagen) and sequenced. CquiOR27 and CquiOR28 were subcloned

113 separately from pGEM-T-CquiOR27 and pGEM-T-CquiOR28 into pGEMHE using In-Fusion

114 HD Cloning Kit according to the manufacturer's instructions (Clontech). PCR In-Fusion primers

115 were designed according to the user manual. CquiOR27-In-Fusion-Fw primer:

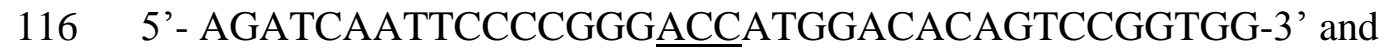

117 CquiOR27-In-Fusion-Rv primer:

118 5'- TCAAGCTTGCTCTAGATTACAACTTGTTAAGCAAATC-3';

119 CquiOR28-In-Fusion-Fw primer:

120 5'- AGATCAATTCCCCGGGACCATGGACGCCACCCAGCGA-3’ and

121 CquiOR28-In-Fusion-Rv primer:

122 5'- TCAAGCTTGCTCTAGATTACAGTTTGCTCAAAACGTC-3’.

123 Kozak sequence ACC (underlined) was added right before the start codon of the target gene. The

124 colonies from transformation were verified by regular PCR with the cloning primers. The positive

125 clones were cultured and subsequently extracted using the QIAprep Spin Miniprep kit (Qiagen)

126 and sequenced by ABI 3730 automated DNA sequencer.

127 To identify introns for qPCR primer design, gDNA was extracted using Wizard Genomic DNA

128 Purification Kit (Promega). Mosquitoes were homogenized individually with $600 \mu$ l of Nucleic

129 Lysis Solution in ice-cold centrifuge tubes and placed at $65^{\circ} \mathrm{C}$ for $30 \mathrm{~min}$. Subsequently, $3 \mu \mathrm{l}$ of

130 RNase was added, the mixture was incubated at $37^{\circ} \mathrm{C}$ for $30 \mathrm{~min}$ and finally cooled to room

131 temperature for $5 \mathrm{~min}$. Then, $200 \mu \mathrm{l}$ of Protein Precipitation Solution was added, incubated on 
132 ice for $5 \mathrm{~min}$, and centrifuged at $14,000 \mathrm{x} \mathrm{g}$ for $4 \mathrm{~min}$. The supernatant was removed and

133 transferred to a new tube containing $600 \mu \mathrm{l}$ of isopropanol. After mixing, inverting, and

134 centrifuging at $14,000 \mathrm{x}$ g for $1 \mathrm{~min}$, the supernatant was removed, and $600 \mu 1$ of $70 \%$ ethanol

135 was added. The gDNA pellet was carefully washed, the tube was centrifuged at 14,000 x $\mathrm{g}$ for 1

$136 \mathrm{~min}$, ethanol was aspirated, and the pellet was air-dried for $15 \mathrm{~min}$. gDNA was reconstituted with

$137100 \mu \mathrm{l}$ of DNA Rehydration Solution at $65^{\circ} \mathrm{C}$, for $1 \mathrm{~h}$. Lastly, gDNA was quantified by

138 NanoDrop ${ }^{\mathrm{TM}}$ Lite Spectrometer (Thermo Fisher Scientific, Waltham, MA), and the quality was

139 verified by agarose gel. The following primers were used to obtain the CquiOR27 gDNA

140 sequence.

141 gCqOR27Fw1: 5'-ATGGACACAGTCCGGTGGG-3'

142

gCqOR27Rv1: 5'-TTACAACTTGTTAAGCAAATCTTTGAGAACC-3'

gCqOR27Fw2: 5'-GGTTGCATATTCGAACAAATTTTGCTCACTATC-3'

146 gCqOR27Rv2: 5'-GATAGTGAGCAAAATTTGTTCGAATATGCAACC-3'

148 PCR products were extracted with Monarch PCR \& DNA Gel Cleanup Kit (New England

149 Biolabs, Ipswich, MA) and sequenced by ABI 3730 automated DNA sequencer.

\section{2.3. qPCR}

152 For quantitative PCR, RNA was extracted from antennal tissues with TRIzol reagent

153 (Invitrogen), and cDNAs were synthesized from $200 \mathrm{ng}$ of RNA of each sample using iScript ${ }^{\mathrm{TM}}$

154 gDNA Clear cDNA Synthesis Kit (Bio-Rad, Hercules, CA) according to the manufacturer's

155 protocol. Real-time quantitative PCR analyses were done by using SYBR ${ }^{\text {TM }}$ Select Master Mix

156 for CFX (appliedbiosystems by Thermo Fisher Scientific), a CFX96 Touch ${ }^{\text {TM }}$ Real-Time PCR 
157 Detection System (Bio-Rad), and having CquiRPS7 gene as a reference. The following primers

158 were designed by the Primer 3 program (https://bioinfo.ut.ee/primer3-0.4.0/). Each forward

159 (left) primer was manually selected to flank splicing junctions to make PCR products only from

160 transcripts.

161 qCqRPS7-F: 5'-ATCCTGGAGCTGGAGATGA-3’

162 pCqRPS7-R: 5'-GATGACGATGGCCTTCTTGT-3'

163 qPCROR32-F: 5'-TCAAAGGGCTACGAATGGTC-3'

164 qPCROR32-R: 5'-GTGCGTCCAATACCGAAAGT-3'

165 qCqOR27-F: 5'-GATTTTTTCCGTGAAGTTGCTATAG-3’

166 qCqOR27-R: 5'-GCCAATGGCTGATCAAAACT-3'

167 qPCR was performed in three biological replicates, each having three technical replicates. Data

168 were analyzed using the $2^{-\Delta \Delta \mathrm{Ct}}$ method. Normalized data are displayed after dividing all 2- $\Delta \Delta \mathrm{Ct}$

169 values by the mean $2^{-\Delta \Delta C t}$ for each group. One group was obtained with 5-day non-blood-fed

170 mosquitoes and the other with 5-days after blood feeding, thus comparing relative expression

171 levels of CquiOR32 and CquiOR27 in blood-seeking and oviposition-ready mosquitoes.

173 2.4. Oocytes preparations and two-electrode voltage-clamp recordings (TEVCs)

174 TEVC was performed as previously described (Leal et al., 2013; Pelletier et al., 2015; Xu et al.,

175 2019; Xu et al., 2014; Xu et al., 2013; Xu et al., 2012a; Xu et al., 2012b; Xu and Leal, 2013; Xu

176 et al., 2020; Xu et al., 2015; Zhu et al., 2013). In brief, linearized pGEMHE-CquiORs were

177 used as templates to transcribe into capped cRNA with poly(A) using an mMESSAGE

178 mMACHINE T7 kit (Ambion, Austin, TX) following the manufacturer's protocol. The cRNAs 
179 were dissolved in RNase-free water and adjusted at a concentration of $200 \mu \mathrm{g} / \mathrm{mL}$ by UV

180 spectrophotometry (NanoDrop ${ }^{\mathrm{TM}}$ Lite Spectrophotometer). $9.2 \mathrm{nl}$ of a mixture of an OR and

181 Orco cRNAs was microinjected into stage V or VI Xenopus oocytes (purchased from EcoCyte

182 Bioscience, Austin, TX). Then, injected oocytes were incubated at $18^{\circ} \mathrm{C}$ for 3-7 days in

183 modified Barth's solution [in mM: $88 \mathrm{NaCl}, 1 \mathrm{KCl}, 2.4 \mathrm{NaHCO}_{3}, 0.82 \mathrm{MgSO}_{4}, 0.33 \mathrm{Ca}\left(\mathrm{NO}_{3}\right)_{2}$,

$1840.41 \mathrm{CaCl}_{2}, 10$ HEPES, pH 7.4] supplemented with $10 \mu \mathrm{g} / \mathrm{mL}$ of gentamycin, $10 \mu \mathrm{g} / \mathrm{mL}$ of

185 streptomycin, and $1.8 \mathrm{mM}$ sodium pyruvate. Test oocytes were placed in a perfusion chamber

186 and challenged with a panel of odorants (see below). Currents were amplified with an OC-725C

187 amplifier (Warner Instruments, Hamden, CT) holding the voltage at $-80 \mathrm{mV}$ and a low-pass

188 filter at $50 \mathrm{~Hz}$, and digitized at $1 \mathrm{kHz}$. Data acquisition and analysis were carried out with

189 Digidata 1440A and pClamp10 software (Molecular Devices, LLC, Sunnyvale, CA).

191 2.4. Panel of odorants

192 The following compounds were used to challenge CquiOR27/CquiOrco- and

193 CquiOR28/CquiOrco-expressing oocytes. Initially, the entire panel was screened with

194 individual compounds at $1 \mathrm{mM}$ diluted in perfusion buffer from their stock $1 \mathrm{M}$ solutions. (-)-

195 Caryophyllene oxide, (-)-menthone, $(+)$-limonene oxide, $(+)$ - $\delta$-cadinene, $( \pm)$-citronellal, $( \pm)-$

196 lactic acid, (士)-linalool, $(E)$-2-heptenal, $(E)$-2-hexenal, $(E)$-2-hexenoic acid, $(E)$-2-hexenyl

197 acetate, $(E)$-2-hexenyl acetate, $(E)$-2-methyl-2-butenal, $(E)$-2-nonenal, $(E)$-3-hexenoic acid, $(E)$ -

198 cinnamaldehyde, $(E, E)$-farnesol, $(E, E)$-farnesyl acetate, $(R)-(+)$-pulegone, $(S)-(-)$ -

199 perillaldehyde, (Z)-3-hexenyl acetate, $(Z)$-8-undecenal, 1,2-dimethoxybenzene, 1,4-

200 diaminobutane, 1,5-diaminopentane, 1-butanol, 1-dodecanal, 1-dodecanol, 1-heptanol, 1- 
201 hepten-3-ol, 1-hexadecanol, 1-hexanol, 1-hexen-3-ol, 1-methylindole, 1-nonanol, 1-octanol, 1-

202 octen-3-ol, 1-octen-3-one, 1-octen-3-yl acetate, 1-octyn-3-ol, 1-pentanol, 1-phenylethanol, 2,3-

203 butanediol, 2,3-butanedione, 2,3-dimethylphenol, 2,4-dimethylphenol, 2,4-hexadienal, 2,4-

204 thiazolinedione, 2,5-dimethylphenol, 2,6-dimethylphenol, 2-acetylthiophene, 2-butanol, 2-

205 butanone, 2-butoxyethanol, 2-ethyltoluol, 2-heptanone, 2-hexanone, 2-hexen-1-ol, 2-methoxy-

206 4-propylphenol, 2-methyl-2-thiazoline, 2-methyl-3-buten-2-ol, 2-methylindole, 2-

207 methylphenol, 2-nonanone, 2-nonen-1-ol, 2-octanol, 2-octanone, 2-oxobutyric acid, 2-

208 oxovaleric acid, 2-pentanol, 2-pentanone, 2-phenoxyethanol, 2-phenylethanol, 2-pyrrolidinone,

209 2-tridecanone, 2-undecanone, 3,4-dimethylphenol, 3,5-dimethylphenol, 3-hexen-1-ol, 3-

210 hydroxy-2-butanone, 3-methyl-1-butanol, 3-methyl-2-butanol, 3-methyl-2-buten-1-ol, 3-

211 methylbenzamide, 3-methylindole (skatole), 3-methylphenol, 3-octanol, 3-octyn-1-ol, 3-

212 pentanol, 4,5-dimethylthiazole, 4-dimethylamino-1-naphthaldehyde, 4-ethylphenol, 4-

213 methylcyclohexanol, 4-methylindole, 4-methylphenol, 5-hexanoic acid, 5-isobutyl-2,3-

214 dimethylpyrazine, 5-methyl-2-hexanone, 5-methylindole, 6-methyl-5-hepten-2-one, 6-

215 methylindole, 7-hydroxcitronellal, 7-methylindole, acetophenone, acetylacetone, benzaldehyde,

216 benzyl alcohol, benzyl formate, butan-2-yl 2-(2-hydroxyethyl)piperidine-1-carboxylate

217 (picaridin), butanal, butanoic acid, butyl acetate, butylamine, cadaverine, camphor, carbon

218 disulfide, carvacrol, carvone, cinnamyl alcohol, citral, citronellol, cyclohexanone, cymene,

219 decanal, decanoic acid, decyl acetate, dibutyl phthalate, dimethyl phthalate, dimethyl trisulfide,

220 dodecanoic acid, ethanoic acid, ethyl 2-(E)-4-(Z)-decadienoate, ethyl 3-hydroxybutanoate, ethyl

221 3-hydroxyhexanoate, ethyl acetate, ethyl butanoate, ethyl hexanoate, ethyl lactate, ethyl

222 linoleate, ethyl phenylacetate, ethyl propionate, ethyl stearate, ethyl $N$-acetyl- $N$-butyl- $\beta$ - 
223 alaninate (IR3535), eucalyptol, eugenol, eugenyl acetate, farnesene, fenchone, furfural,

224 geraniol, geranyl acetate, geranylacetone, guaiacol, heptanal, heptanoic acid, heptyl acetate,

225 heptylamine, hexanal, hexanoic acid, hexyl acetate, hexylamine, indole, isobutyric acid,

226 isopentyl acetate, isoprene, isopropyl myristate, isovaleraldehyde, isovaleric acid, jasmone,

227 limonene, linalool oxide, linalyl acetate, linoleic acid, menthol, menthyl acetate, methyl $(N, N)$ -

228 dimethylanthranilate, methyl acetate, methyl anthranilate, methyl butyrate, methyl disulfide,

229 methyl hexanoate, methyl myristate, methyl propionate, methyl salicylate, m-toluamide,

230 myristic acid, $N$-(2-isopropylphenyl)-3-methylbenzamide, $N$-(sec-butyl)-2-methylbenzamide,

$231 N, N$-diethyl-3-methylbenzamide (DEET), nerol, nerolidol, $N$-methylbenzamide, nonanal,

232 nonanoic acid, nonyl acetate, $N$-sec-butyl-2-phenyl-acetamide, n-tridecanoic acid, ocimene,

233 octadecyl acetate, octanal, octanoic acid, octyl acetate, octylamine, oleic acid, palmitic acid

234 methyl ester, palmitoleic acid, p-coumaric acid, penatanal, pentanoic acid, pentyl acetate,

235 pentylamine, phenethyl formate, phenethyl propionate, phenol, phenyl isobutyrate, phenyl

236 propanoate, phenylacetaldehyde, phenylether, p-menthane-3,8-diol (PMD), propanal, propanoic

237 acid, propyl acetate, propylamine, pyridine, pyrrolidine, sabinene, $\beta$-caryophyphyllene, $\beta$ -

238 myrcene, terpinen-4-ol, terpinolene, thujone, thymol, trimethylamine, trimethylamine,

239 undecanal, $\alpha$-hexylcinnamaldehyde, $\alpha$-humulene, $\alpha$-methylcinnamaldehyde, $\alpha$ -

240 methylcinnamaldehyde, $\alpha$-phellandrene, $\alpha$-pinene, $\alpha$-terpinene, $\alpha$-terpineol, $\gamma$-decalactone, $\gamma$ -

241 dodecalactone, $\gamma$-hexalactone, $\gamma$-octalactone, $\gamma$-terpinene, and $\gamma$-valerolactone. The Orco ligand

242 candidate 2-\{[4-Ethyl-5-(4-pyridinyl)-4H-1,2,4-triazol-3-yl]sulfanyl $\}-N$-(4-

243 isopropylphenyl)acetamide (OLC 12 = VUAA-3) was used for confirming receptor protein

244 expression. 
246 2.5. Surface landing and feeding and hand-in cage assays.

247 Repellency was measured using a surface landing and feeding assay described in detail

248 elsewhere (Leal et al., 2017; Xu et al., 2014), with a slight modification. In short, this dual-

249 choice assay had two Duddle bubbling tubes, which protrude from a wooden board inside of the

250 arena (mosquito cage). Water was circulated inside the Duddle tubes to maintain their surface

251 temperature at $37^{\circ} \mathrm{C}$. A needle was placed on the top of each Duddle tube with the dual function

252 of holding dental cotton rolls and delivering $\mathrm{CO}_{2}$ at $50 \mathrm{ml} / \mathrm{min}$. Defibrinated sheep blood (100

$253 \mu \mathrm{l})$ was applied to each cotton roll. To avoid contamination of the tubes with mosquito

254 excretion, each tube was covered with a non-lubricated condom (Trojan ENZ) and replaced

255 after five tests. Each Duddle tube was surrounded by a filter paper cylinder to which $200 \mu \mathrm{l}$ of a

256 test repellent solution or solvent only (control) was applied. Thus, one side of the arena (test)

257 had a chemical curtain providing spatial repellency. Test mosquitoes (100 non-blood-fed, at

258 least two weeks old) were deprived of water and sugar for at least one hour before the assays.

259 DEET (1\%) was used as a standard for comparison. Repellency tests were performed by testing

260 DEET at one side of the arena, then DEET at the opposite side, two replicates of a test

261 compound, one at each side of the arena, and continuing in this order for two or three complete

262 cycles. Behavioral responses were expressed in protection rate according to WHO (WHO,

263 2009) and EPA (EPA, 2010) recommendations: $\mathrm{P} \%=(\mathrm{C}-\mathrm{T}) / \mathrm{C}) \mathrm{X} 100$, where $\mathrm{T}$ and $\mathrm{C}$ represent

264 the number of mosquitoes in the treatment and control sides of the arena after 5 min.

265 The hand-in cage assay is a simplified version of the standard WHO (WHO, 2009) and EPA

266 (EPA, 2010) arm-in cage assay. The four major differences are the cage's size, hands vs. arm, 
267 the duration of the exposure, and starvation time: $1 \mathrm{~h}$ in vs. 12-24 $\mathrm{h}$ in WHO (WHO, 2009) and

268 EPA (EPA, 2010) protocols. Collapsible field cages $(30$ x 30 x 30 cm; BioQuip Products,

269 Rancho Cordova, CA) were used. One hundred to 200 non-blood-fed, 10-14 days old

270 mosquitoes were used per cage. The test subject (W.S.L.) inserted a hand, which was covered

271 with a glove, thus exposing only four fingers (see below). With a smaller cage, mosquitoes

272 showed a robust response in a shorter time $(1.5 \mathrm{~min})$ than the duration recommended by WHO

273 (WHO, 2009) and EPA (EPA, 2010), i.e., 3 and $5 \mathrm{~min}$, respectively. Mosquitoes landing on the

274 subject fingers for longer than $3 \mathrm{~s}$ were gently removed with a soft brush to prevent feeding.

275 The UC Davis IRB was consulted and determined that IRB review was not required.

277 2.6. Formulation of $\gamma$-octalactone for the hand-in cage assay

278 A $\gamma$-octalactone cream formulation was prepared by adding stearyl alcohol $(1.25 \mathrm{~g})$ to $15 \mathrm{ml}$ of

279 water in a 50-ml beaker on a stirring hot plate and stirred with a magnetic bar until the alcohol

280 melted. Next, $1.25 \mathrm{~g}$ of cetearyl alcohol was added and melted before adding $\gamma$-octalactone

281 (1.75 g). Subsequently, polysorbate 80 (1.25 g), sorbitol oleate (1.25 g), stearic acid (1.25 g),

282 glyceryl stearate self-emulsifying (1.25 g), and C12-C15 alkyl benzoate ( $0.5 \mathrm{~g})$ were added in

283 tandem after waiting to dissolve or melt. After $5 \mathrm{~min}$, the mixture was cooled to $50^{\circ} \mathrm{C}$ before

284 adding $0.25 \mathrm{ml}$ of cyclomethicone. The resulting mixture was then transferred to a $50-\mathrm{ml}$

285 conical centrifugal tube and let cool down to room temperature for subsequent use. For control,

286 a similar cream formulation was prepared without $\gamma$-octalactone. The $\gamma$-octalactone cream

287 formulation (ca. $1 \mathrm{~g}$ ) was applied to the right hand's small, ring, middle, and index fingers. The

288 same amount of the control cream formulation was similarly applied to the left hand. Both 
289 hands were covered with Nitri-Solve 730-20 gloves, which were prepared by cutting the finger

290 covers (except the thumbs), leaving only ca. $2 \mathrm{~cm}$ covering the proximal phalanx. Thus, the

291 treated area had ca. $0.4 \mathrm{mg}$ of $\gamma$-octalactone $/ \mathrm{cm}^{2}$.

292

\subsection{Statistical analysis and graphical preparations}

294 Prism 9.3.1 from GraphPad Software (La Joya, CA) was used for both statistical analysis and

295 graphical preparations. A dataset that passed the Shapiro-Wilk normality test was analyzed by t-

296 test; otherwise, data were analyzed by Wilcoxon matched-pairs signed-rank or Mann-Whitney

297 test, as specified below. All data are presented as mean \pm SEM.

\section{Results and discussion}

300 3.1. CquiOR32/CquiOR27 relative expression

301 We cloned the full-length cDNA of CquiOR27 (GenBank OM240661) using primers designed

302 based on a previously reported DNA sequence (Taparia et al., 2017). To design qPCR primers

303 flanking splicing junctions, we cloned CquiOR27 gDNA (GenBank OM240662), given that the

304 predicted RNA/mRNA sequence was not found in VectorBase. We re-analyzed our

305 transcriptome data (Leal et al., 2013) and observed that TCONS_00034458 (Table S02) (Leal et

306 al., 2013) is 97\% identical to a segment (61\%) of CquiOR27 DNA, with a 1\% gap. We then

307 compared by qPCR the relative expression levels of CquiOR32 and CquiOR27 at two different

308 physiological stages, i.e., 5 days-old, non-blood-fed (host-seeking mosquitoes) and 5 days post

309 blood feeding (oviposition-ready mosquitoes). qPCR analyses of CquiOR28 were not

310 performed because the receptor did not respond to any test compounds (see below). Although 
311 the transcription levels of the previously reported "inhibitory receptor" CquiOR32 were higher

312 than that of CquiOR27 in both physiological stages (Fig. 1), CquiOR27 showed relatively

313 higher expression levels in non-blood-fed mosquitoes consistent with a possible role in host

314 finding.

316 3.2. CquiOR27 generated regular and inhibitory currents

317 To deorphanize CquiOR27, we used a panel of 250 odorants and two-electrode voltage-clamp

318 recordings from Xenopus laevis oocytes co-expressing CquiOR27 and CquiOrco. Small and

319 moderate inward currents were elicited by fenchone, citronellal, alpha-terpineol, $N$-sec-butyl-2-

320 phenyl-acetamide, camphor, ethyl linoleate, 1-phenylethanol, 2,3-dimethylphenol, 3,5-

321 dimethylphenol, jasmone, octadecyl acetate, limonene oxide, carvone, $(R)-(+)$-pulegone, and

322 menthyl acetate. Additionally, three compounds, linalool oxide, $\gamma$-octalactone, and $\gamma$ -

323 decalactone, elicited robust responses (Fig. 2). By contrast, 2,6-dimethylphenol, 3,4-

324 dimethylphenol, 2,4-dimethylphenol, 2,3-dimethylphenol generated inhibitory currents,

325 although the strongest responses were elicited by octylamine (Fig. 2). Of note, two phenolic

326 compounds (2,3-dimethylphenol and 3,5-dimethylphenol) elicited inward currents, whereas

327 others showed inhibitory responses.

328 Although CquiOR27 showed a promiscuous profile, $\gamma$-octalactone and $\gamma$-decalactone were by

329 far the best ligands in our screenings (Fig. 3), with a moderate response to linalool oxide.

330 Interestingly, the responses to $\gamma$-hexalactone and $\gamma$-dodecalactone were very small. The dose-

331 response relationship confirmed that indeed $\gamma$-octalactone was the best ligand (Fig. 4). 
333 3.3. Intrarecptor inhibition manifested in the Xenopus oocytes recording system

334 We challenged CquiOR27/CquiOrco-expressing oocytes with excitatory and inhibitory

335 compounds. Specifically, we presented $\gamma$-octalactone, octylamine, and a mixture of the two

336 compounds. Octylamine per se generated strong inhibitory currents (upward deflections),

337 whereas $\gamma$-octalactone alone gave robust stimulatory currents. The $\gamma$-octalactone-generated

338 inward currents decreased significantly in a dose-dependent manner when this odorant was co-

339 applied with octylamine (Fig. 5). Oocyte adaptation was ruled out as there was no significant

340 difference between the $\gamma$-octalactone-elicited responses before and after co-applications with

341 octylamine ( $\mathrm{p}=0.9683$, Mann-Whitney test). Similar findings were observed when 2,3-

342 dimethylphenol was used as the inhibitory compound (Figs. 1). We, therefore, concluded that

343 both stimulatory and inhibitory compounds are acting on the same CquiOR27/CquiOrco

344 complex, thus suggesting an intrareceptor interaction.

345 As previously discussed (Xu et al., 2019), there are at least two possible explanations for the

346 currents in the reverse direction elicited by octylamine and other inhibitory compounds. These

347 ligands may be negative allosteric inhibitors, or perhaps they are inverse agonists. It is

348 conceivable that by binding to CquiOr27/CquiOrco-complex, the open $<=>$ close receptor

349 equilibrium shifts towards the close, inactive conformation, thus suppressing signaling. In the

350 case of CquiOR32 (Xu et al., 2019), we reasoned that, given the different structures,

351 stimulatory and inhibitory compounds might be acting on different binding sites. This argument

352 is still valid when comparing $\gamma$-octalactone with octylamine. However, the observation that

353 isomers of dimethylphenol gave inward and outward currents suggests that the compounds

354 eliciting currents in the reverse direction might be inverse agonists. 
356 3.4. CquiOR28 is non-functional

357 We selected and sequenced 32 colonies of CquiOR28. They gave four variants (OM258698-

358 OM258701), which were subcloned in pGEMHE and, subsequently, tested in the Xenopus

359 oocyte recording system. No stimulatory or inhibitory currents were recorded when these

360 variants were challenged with our panel of odorants. Because none of the variants responded to

361 Orco ligand candidates (VUAA-1 or OCL12), we concluded that CquiOR28 is not a functional

362 receptor.

363

364 3.5. $\gamma$-Octalactone is a potent repellent

365 Given that $\gamma$-dodecalactone and related compounds have been previously reported as mosquito

366 repellents (Bedoukian, 2016), we tested the repellency activity of the major ligands for

367 CquiOR27 against Cx. quinquefasciatus. Specifically, we measured $\gamma$-octalactone and $\gamma$ -

368 decalactone repellency against $C x$. quinquefasciatus using the surface landing and feeding assay

369 (Leal et al., 2017; Xu et al., 2014) and compared their potency with the gold standard of insect

370 repellents, DEET. Repellency activity was expressed in protection, according to WHO (WHO,

$3712009)$ and EPA (EPA, 2010) recommendations. Of note, protection (P) differs slightly from the

372 repellency index (RI) commonly used in insect behavior. These indexes are obtained by

373 dividing the responses to (control minus treatment) by the responses to control only (WHO and

374 EPA protocol) or (control plus treatment) in the generic repellency index. Specifically, $\mathrm{P}=(\mathrm{C}-$

$375 \mathrm{~T}) / \mathrm{C}$ and $\mathrm{RI}=(\mathrm{C}-\mathrm{T}) /(\mathrm{C}+\mathrm{T})$, respectively. Thus, when $\mathrm{T}$ is very small, as in this case, the two

376 indexes are nearly equal. 
377 The protection elicited by $1 \% \gamma$-octalactone $(91.2 \pm 2.3 \%)$ was not significantly different from

378 the protection obtained with the same dose of DEET $(90.0 \pm 2.8 \%) \mathrm{p}=0.2416$, Wilcoxon

379 matched-pairs signed-rank test). Similarly, $\gamma$-decalactone $(85.9 \pm 3.5 \%)$ did not differ

380 significantly from DEET $(85.0 \pm 2.1 \%, \mathrm{p}=0.8438)$. We then directly compared $\gamma$-octalactone

381 with $\gamma$-decalactone by measuring their repellency with the same test mosquitoes. The protection

382 elicited by $\gamma$-octalactone $(89.0 \pm 2.6 \%)$ was not significantly different from that measured with

$383 \gamma$-decalactone $(89.3 \pm 2.6 \%, \mathrm{~N}=25, \mathrm{p}=0.9935$, Figs 2$)$. In short, $\gamma$-octalactone did not differ

384 significantly from $\gamma$-decalactone, and these two natural repellents did not differ from DEET in

385 our assays. By contrast, $\gamma$-dodecalactone $(65.7 \pm 6.1 \%)$ showed a significantly lower repellency

386 activity than DEET $(85.8 \pm 4.6 \%, \mathrm{~N}=10, \mathrm{p}=0.0171$, paired, two-tailed t-test $)$.

387 Previously, delta-octalactone has been reported as a repellent against the tsetse fly, Glossina

388 morsitans (Mwangi et al., 2008). Still, a literature survey (PubMed and Google Scholar) did not

389 yield any evidence that $\gamma$-octalactone has been previously reported as a mosquito repellent. This

390 is surprising because of the extensive prospect of repellent from natural sources (Gross and

391 Coats, 2014; Paluch et al., 2010).

392 Given the strong responses observed in the surface landing and feeding assay, we next tested $\gamma$ -

393 octalactone in a modified hand-in-cage assay. We designed a simplified version of the arm-in-

394 cage assay recommended by WHO (WHO, 2009) and EPA (EPA, 2010). With a smaller cage,

395 mosquitoes readily landed on the untreated subject's fingers, thus allowing a shorter (1.5 min)

396 testing time. For these hand-in cage assays, we used a 7\% cream formulation, consistent with

397 the lowest percentage of DEET products in the market. Mosquito responses to one hand of the

398 subject treated with a $\gamma$-octalactone cream formulation were compared with the responses to the 
399 other hand covered with a control cream devoid of $\gamma$-octalactone (untreated). $\gamma$-Octalactone

400 provided $97.0 \pm 1.3 \%$ protection (Fig. 6 ), with $47.6 \pm 8.3 \%$ and $1.3 \pm .7 \%$ landings per trial in

401 the untreated and treated hands, respectively $(\mathrm{N}=8, \mathrm{p}=0.0078$, Wilcoxon matched-pairs signed-

402 rank test).

403

404 3.4. Overall conclusions

405 Our reverse chemical ecology approach led to discovering a potent spatial natural repellent of

406 potential practical applications. We showed that $\gamma$-octalactone is a strong repellent, but it is

407 necessary to develop a slow-release formulation for complete long-term protection. Of note, $\gamma$ -

408 octalactone is a natural compound found in peaches, mangoes, beef, and ham (PubChem) used

409 in hand creams and as a flavoring agent.

410 We found evidence in these studies that, like CquiOR32 (Xu et al., 2019), CquiOr27 is a

411 receptor with dual current directions (“bidirectional”). Specifically, CquiOR27/CquiOrco-

412 expressing oocytes were activated by some compounds, including $\gamma$-octalactone, and inhibited

413 by other ligands, including octylamine. It is conceivable that the observed currents in the

414 reverse direction, elicited by octylamine and some phenolic compounds, are not outward

415 currents sensu stricto. The observed currents in the reverse direction could be derived from

416 "baseline shift" due to the closing of ion channels (reduction of spontaneous activity) upon

417 CquiOR27 binding to inhibitory compounds. To be rigorously tested, these hypotheses must

418 await the elucidation of the 3D structures of these receptors. 


\section{$420 \quad$ Funding}

421 Research reported in this publication was supported by the National Institute of Allergy and

422 Infectious Diseases (NIAID) of the National Institutes of Health under award number

423 R01AI095514. The content is solely the responsibility of the authors and does not necessarily

424 represent the official views of the NIH.

\section{Author contributions}

427 P.X., Y-M. C., S. A., G.M.L. and W.S.L. performed research; W.S.L. designed research; P.X.

428 and WS.L. analyzed the data; W.S.L. wrote the paper; all authors read and approved the final 429 manuscript.

\section{Competing financial interest}

432 The authors declare no conflict of interest.

\section{Acknowledgments}

435 We thank Erica A. Littman for the preliminary $\gamma$-octalactone repellency tests. The Orco ligand

436 candidate OCL12 was provided by the Vanderbilt Institute of Chemical Biology, Chemical

437 Synthesis Core, Vanderbilt University, Nashville, TN 37232-0412. 
439 Supplementary information

440 Supplementary figures: Figs1 and Figs2

\section{References}

444 Bedoukian, R.H., 2016. Control and repellency of mosquitoes. Google Patents.

445 Chen, S., Luetje, C.W., 2012. Identification of new agonists and antagonists of the insect

446 odorant receptor co-receptor subunit. PloS one 7, e36784.

447 Choo, Y.M., Xu, P., Hwang, J.K., Zeng, F., Tan, K., Bhagavathy, G., Chauhan, K., Leal, W.S., 448 2018. Reverse chemical ecology approach for the identification of an oviposition attractant for 449 Culex quinquefasciatus. Proc Natl Acad Sci U S A in press.

450 de Brito Sanchez, M.G., Kaissling, K.E., 2005. Inhibitory and excitatory effects of iodobenzene 451 on the antennal benzoic acid receptor cells of the female silk moth Bombyx mori L. Chem 452 Senses 30, 435-442.

453 EPA, 2010. Product Performance Test Guidelines OPPTS 810.3700: Insect Repellents to be 454 Applied to Human Skin.

455 Gross, A.D., Coats, J.R., 2014. Can green chemistry provide effective repellents? , in: Debboun, 456 M., Frances, S., Strickman, D. (Eds.), Handbook of Insect Repellents. Taylor \& Francis Group, 457 Boca Raton, FL, p. 383.

458 Kaissling, K.E., 2014. Pheromone Reception in Insects: The Example of Silk Moths, in:

459 Mucignat-Caretta, C. (Ed.), Neurobiology of Chemical Communication, Boca Raton (FL).

460 Leal, W.S., 2005. Pheromone reception. Top Curr Chem 240, 1-36.

461 Leal, W.S., Barbosa, R.M., Zeng, F., Faierstein, G.B., Tan, K., Paiva, M.H., Guedes, D.R., 462 Crespo, M.M., Ayres, C.F., 2017. Does Zika virus infection affect mosquito response to 463 repellents? Sci Rep 7, 42826. 
464 Leal, W.S., Choo, Y.M., Xu, P., da Silva, C.S., Ueira-Vieira, C., 2013. Differential expression

465 of olfactory genes in the southern house mosquito and insights into unique odorant receptor

466 gene isoforms. Proceedings of the National Academy of Sciences of the United States of

467 America 110, 18704-18709.

468 Mwangi, M.T., Gikonyo, N.K., Ndiege, I.O., 2008. Repellent properties of $\delta$-octalactone

469 against the tsetse fly, Glossina morsitans morsitans. Journal of Insect Science 8.

470 Nikonov, A.A., Leal, W.S., 2002. Peripheral coding of sex pheromone and a behavioral

471 antagonist in the Japanese beetle, Popillia japonica. Journal of chemical ecology 28, 1075-

4721089.

473 Paluch, G., Bartholomay, L., Coats, J., 2010. Mosquito repellents: a review of chemical

474 structure diversity and olfaction. Pest Manag Sci 66, 925-935.

475 Pelletier, J., Xu, P., Yoon, K.S., Clark, J.M., Leal, W.S., 2015. Odorant receptor-based

476 discovery of natural repellents of human lice. Insect biochemistry and molecular biology 66,

477 103-109.

478 Su, C.Y., Menuz, K., Reisert, J., Carlson, J.R., 2012. Non-synaptic inhibition between grouped

479 neurons in an olfactory circuit. Nature 492, 66-71.

480 Taparia, T., Ignell, R., Hill, S.R., 2017. Blood meal induced regulation of the chemosensory

481 gene repertoire in the southern house mosquito. BMC Genomics 18, 393.

482 Tauxe, G.M., MacWilliam, D., Boyle, S.M., Guda, T., Ray, A., 2013. Targeting a dual detector

483 of skin and CO2 to modify mosquito host seeking. Cell 155, 1365-1379.

484 Taylor, R.W., Romaine, I.M., Liu, C., Murthi, P., Jones, P.L., Waterson, A.G., Sulikowski, 485 G.A., Zwiebel, L.J., 2012. Structure-activity relationship of a broad-spectrum insect odorant 486 receptor agonist. ACS Chem Biol 7, 1647-1652.

487 WHO, 2009. Guidelines for efficacy testing of mosquito repellents for human skin.

488 Xu, P., Choo, Y.M., Chen, Z., Zeng, F., Tan, K., Chen, T.Y., Cornel, A.J., Liu, N., Leal, W.S., 489 2019. Odorant inhibition in mosquito olfaction. iScience 19, 25-38.

490 Xu, P., Choo, Y.M., De La Rosa, A., Leal, W.S., 2014. Mosquito odorant receptor for DEET

491 and methyl jasmonate. Proceedings of the National Academy of Sciences of the United States

492 of America 111, 16592-16597. 
493 Xu, P., Choo, Y.M., Pelletier, J., Sujimoto, F.R., Hughes, D.T., Zhu, F., Atungulu, E., Cornel, 494 A.J., Luetje, C.W., Leal, W.S., 2013. Silent, generic and plant kairomone sensitive odorant 495 receptors from the Southern house mosquito. Journal of insect physiology 59, 961-966.

496 Xu, P., Garczynski, S.F., Atungulu, E., Syed, Z., Choo, Y.M., Vidal, D.M., Zitelli, C.H., Leal, 497 W.S., 2012a. Moth sex pheromone receptors and deceitful parapheromones. PloS one 7, 498 e41653.

499 Xu, P., Hooper, A.M., Pickett, J.A., Leal, W.S., 2012b. Specificity determinants of the 500 silkworm moth sex pheromone. PloS one 7, e44190.

501 Xu, P., Leal, W.S., 2013. Probing insect odorant receptors with their cognate ligands: insights 502 into structural features. Biochemical and biophysical research communications 435, 477-482.

503 Xu, P., Wen, X., Leal, W.S., 2020. CO2 per se activates carbon dioxide receptors. Insect 504 biochemistry and molecular biology 117, 103284.

505 Xu, P., Zhu, F., Buss, G.K., Leal, W.S., 2015. 1-Octen-3-ol - the attractant that repels. 506 F1000Research 4, 156.

507 Zhu, F., Xu, P., Barbosa, R.M., Choo, Y.M., Leal, W.S., 2013. RNAi-based demonstration of 508 direct link between specific odorant receptors and mosquito oviposition behavior. Insect 509 biochemistry and molecular biology 43, 916-923.

\section{$513 \quad$ Figure legends}

515 Fig. 1. Relative transcript levels of CquiOR32 and CquiOR27

516 Relative gene expression levels of CquiOR32 and CquiOR27 in host-seeking and oviposition-

517 ready mosquitoes. 5D, 5 days; NBF, non-blood-fed; ABM, after a blood meal. 
519 Fig. 2. Currents recorded from CquiOR27/CquiOrco-expressing oocytes with stimulatory

520 and inhibitory compounds

521 Representative trace of the responses elicited by three main stimulatory and one inhibitory

522 compounds, i.e., linalool oxide, $\gamma$-octalactone, $\gamma$-decalactone, and octylamine. This trace was

523 colored to match the colors of the corresponding structures. Dose increased from left to right,

524 from 0.01 to $1 \mathrm{mM}$.

525

526 Fig. 3. Currents recorded from CquiOR27/CquiOrco-expressing oocytes with lactones

527 Recordings obtained with five $\gamma$-lactones at the same dose $(1 \mathrm{mM})$, with the same oocyte

528 preparation.

530 Fig. 4. Concentration-dependent curves obtained with the main stimulatory and

531 inhibitory compounds.

532 Recordings were obtained with five CquiOR27/CquiOrco-expressing oocytes ( $=5)$. Each

533 oocyte was challenged with all four compounds starting with a dose of $10^{-6} \mathrm{M}(1 \mu \mathrm{M})$ and

534 increasing to $10^{-3} \mathrm{M}(1 \mathrm{mM})$. The best ligands were $\gamma$-octalactone $\left(\mathrm{EC}_{50} 4.1 \mathrm{X} 10^{-5} \mathrm{M}\right)$ and $\gamma$ -

535 decalactone $\left(\mathrm{EC}_{50} 5.3 \times 10^{-5} \mathrm{M}\right)$.

537 Fig. 5. Quantification of responses to binary mixtures of stimulatory and inhibitory

\section{8 compounds}

539 Quantification of responses recorded from CquiOR27/CquiOrco-expressing oocytes when

540 challenged with octylamine and $\gamma$-octalactone separately or combined. Each oocyte was 
541 challenged with the inhibitory compound at $1 \mathrm{mM}, \gamma$-octalactone at $0.1 \mu \mathrm{M}$ and then a mixture

542 of $\gamma$-octalactone at $0.1 \mu \mathrm{M}$ and the inhibitory compound in decreasing doses from $1 \mathrm{mM}$ to 0.01

$543 \mathrm{mM}$ and finally $\gamma$-octalactone at $0.1 \mu \mathrm{M}$. Recordings were obtained with 5

544 CquiOR27/CquiOrco-expressing oocytes.

546 Fig. 6. Behavioral responses of $C x$. quinquefasciatus mosquitoes in repellency assays

547 Comparative responses of non-blood-fed female mosquitoes to DEET, $\gamma$-octalactone, $\gamma$ -

548 decalactone, and $\gamma$-dodecalactone in the surface landing and feeding assay. Repellency

549 activities are expressed in the protection rate. The repellency activities of $\gamma$-octalactone (A) and

$550 \gamma$-decalactone (B) did not differ significantly from that of DEET, but $\gamma$-dodecalactone showed

551 significantly lower protection than $\operatorname{DEET}(\mathrm{C})$.

553 Fig. 7. $\boldsymbol{\gamma}$-Octalactone repellency measured in a hand-in cage assay

554 Responses of mosquitoes (landing/probing) to hands covered with a $\gamma$-octalactone cream

555 formulation and a control formulation devoid of $\gamma$-octalactone (untreated).

557 Supplementary figure legends

559 Figs1. Quantification of responses to binary mixtures of stimulatory and inhibitory

560 compounds

561 Quantification of responses recorded from CquiOR27/CquiOrco-expressing oocytes when

562 challenged with 2,3-dimethylphenol and $\gamma$-octalactone separately or combined. Each oocyte 
563 was challenged with the inhibitory compound at $1 \mathrm{mM}, \gamma$-octalactone at $0.1 \mu \mathrm{M}$ and then a

564 mixture of $\gamma$-octalactone at $0.1 \mu \mathrm{M}$ and the inhibitory compound in doses from $1 \mathrm{mM}$ to 0.01

$565 \mathrm{mM}$ and finally $\gamma$-octalactone at $0.1 \mu \mathrm{M}$. Recordings were obtained with 3

566 CquiOR27/CquiOrco-expressing oocytes.

567

568 Figs2. Direct comparison of protection provided by $\boldsymbol{\gamma}$-octalactone and $\boldsymbol{\gamma}$-decalactone

569 There was no significant difference between the repellence activities of $\gamma$-octalactone and $\gamma$ -

570 decalactone as measured by direct comparison in the surface landing and feeding assay $(\mathrm{N}=25$,

$571 \mathrm{P}=0.9935$, Wilcoxon, two-tailed, matched-pairs signed-rank test. 

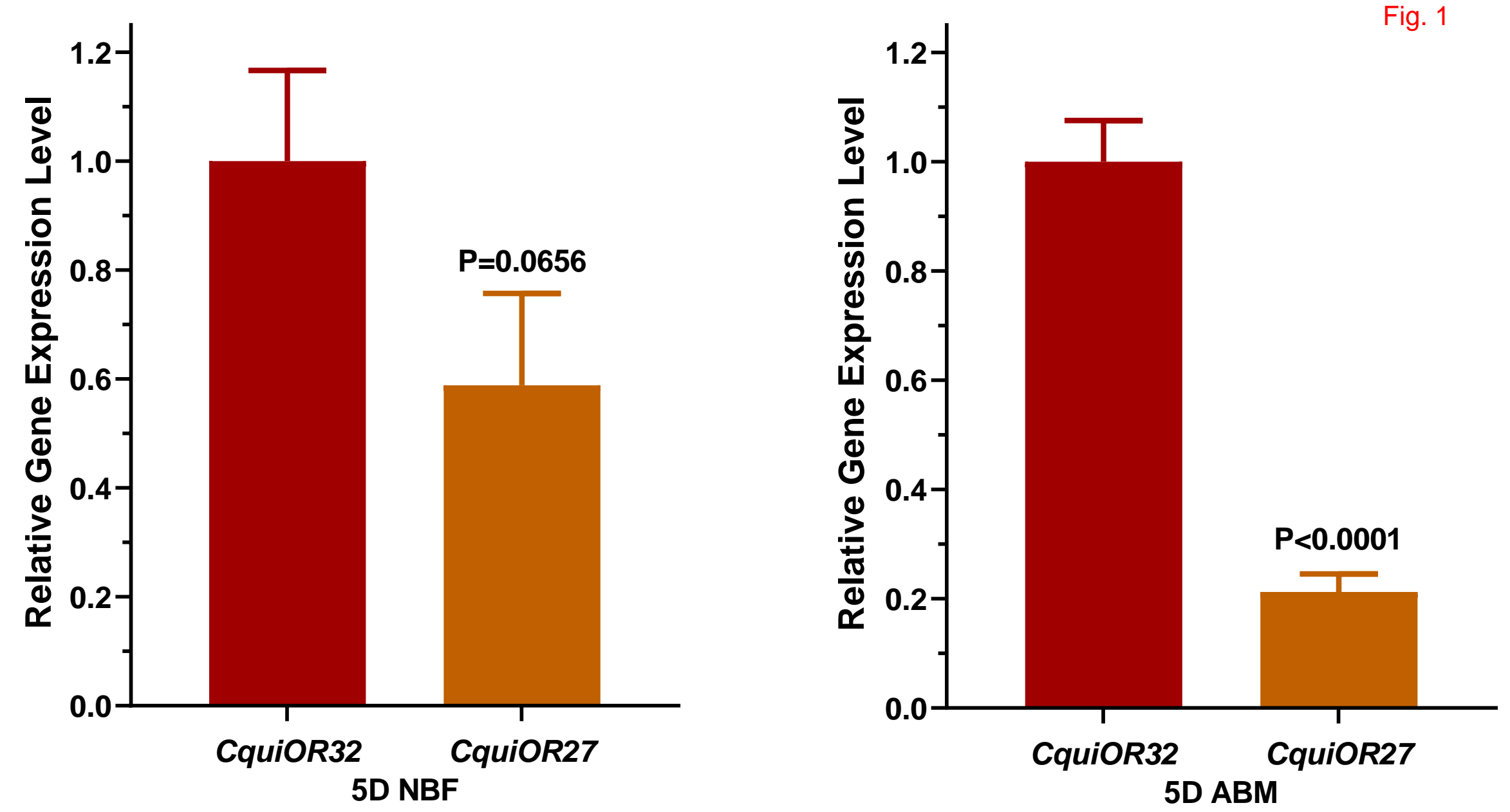


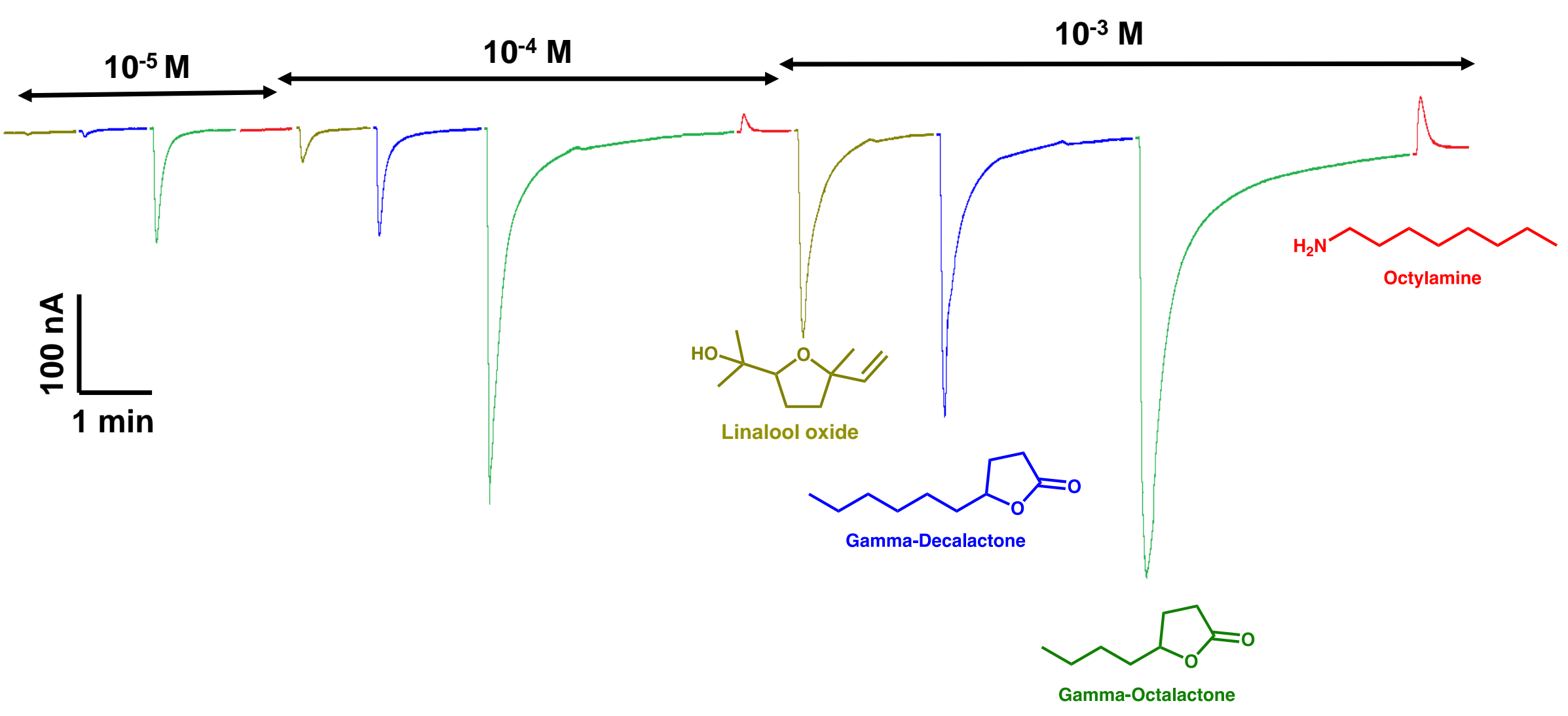




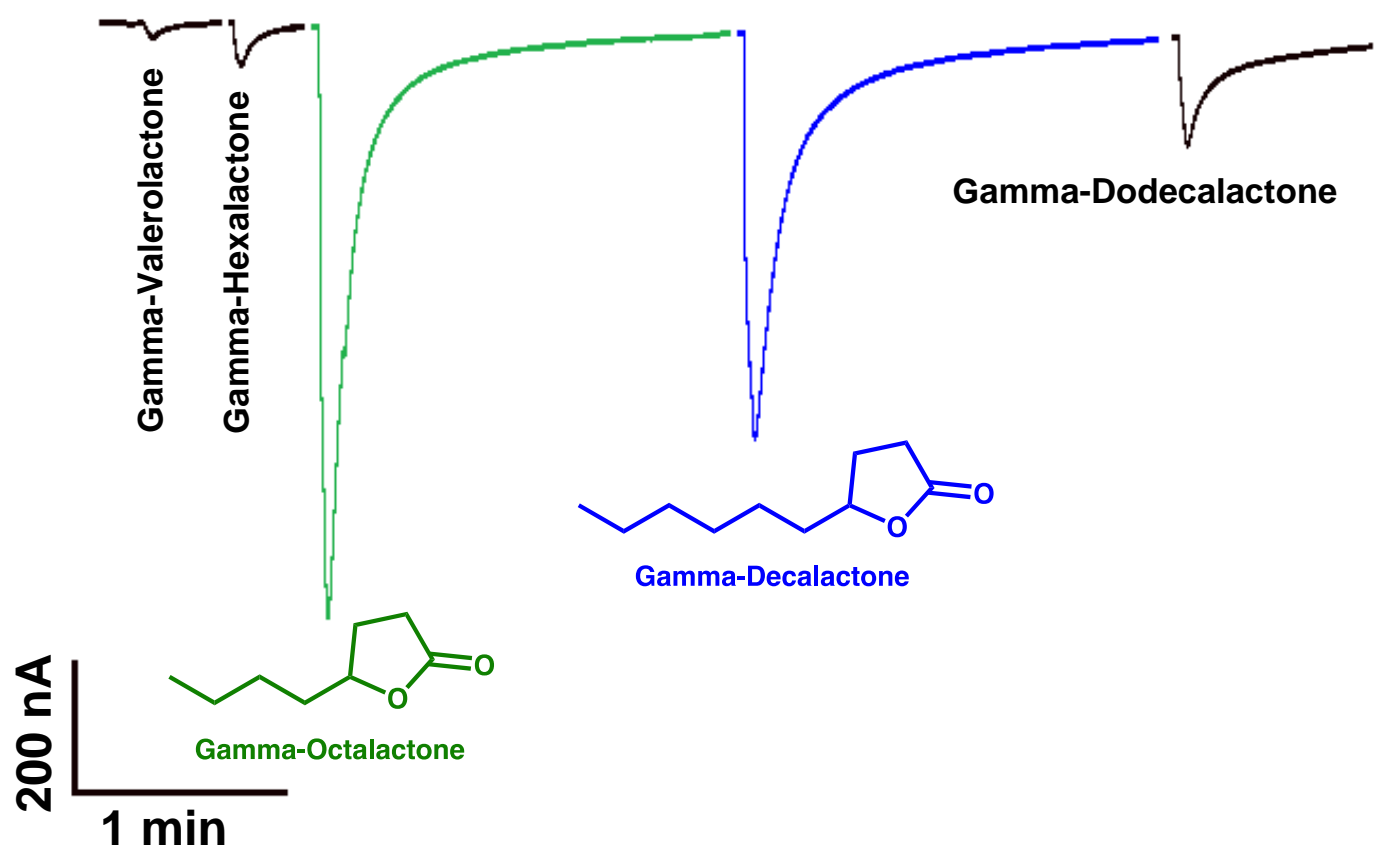




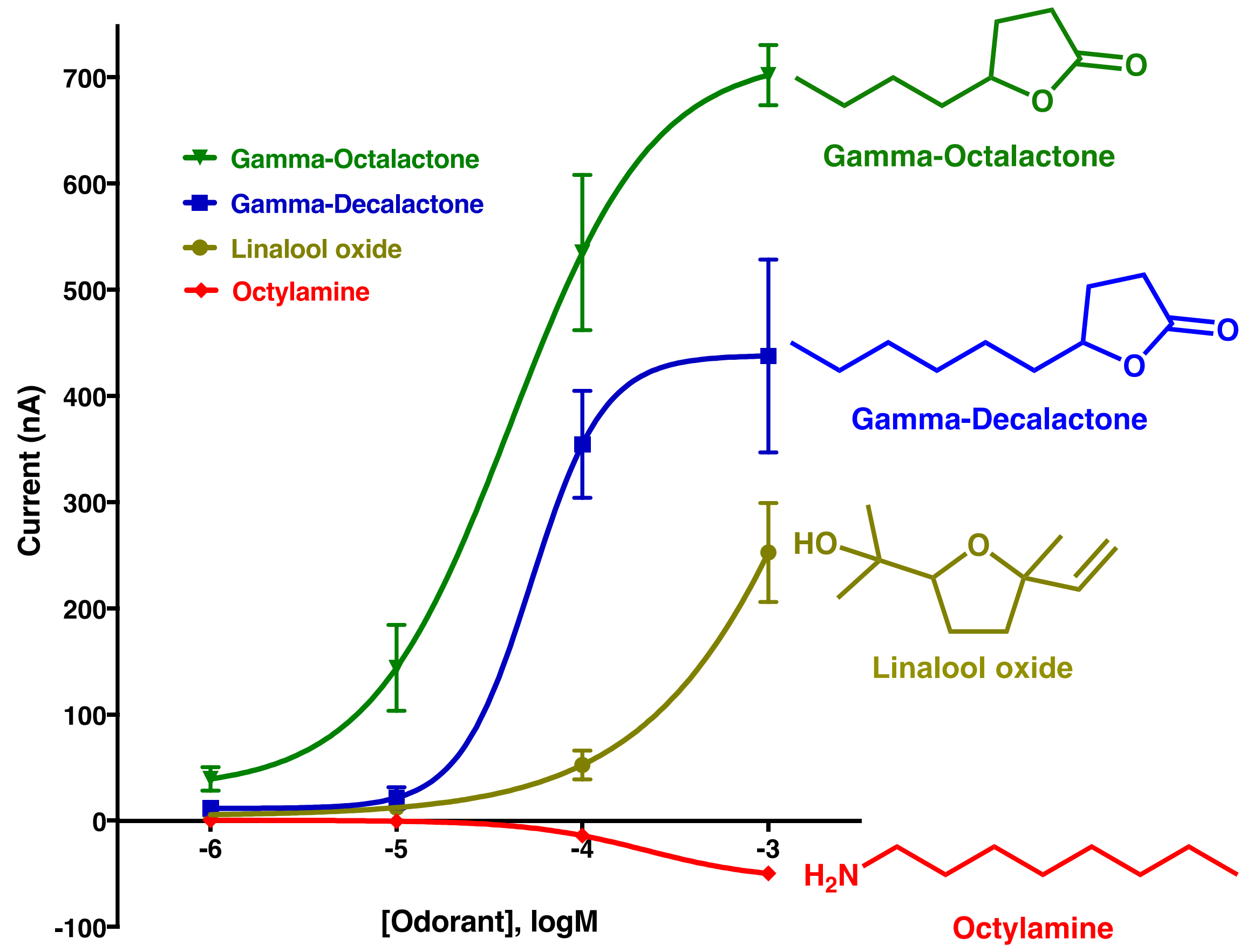




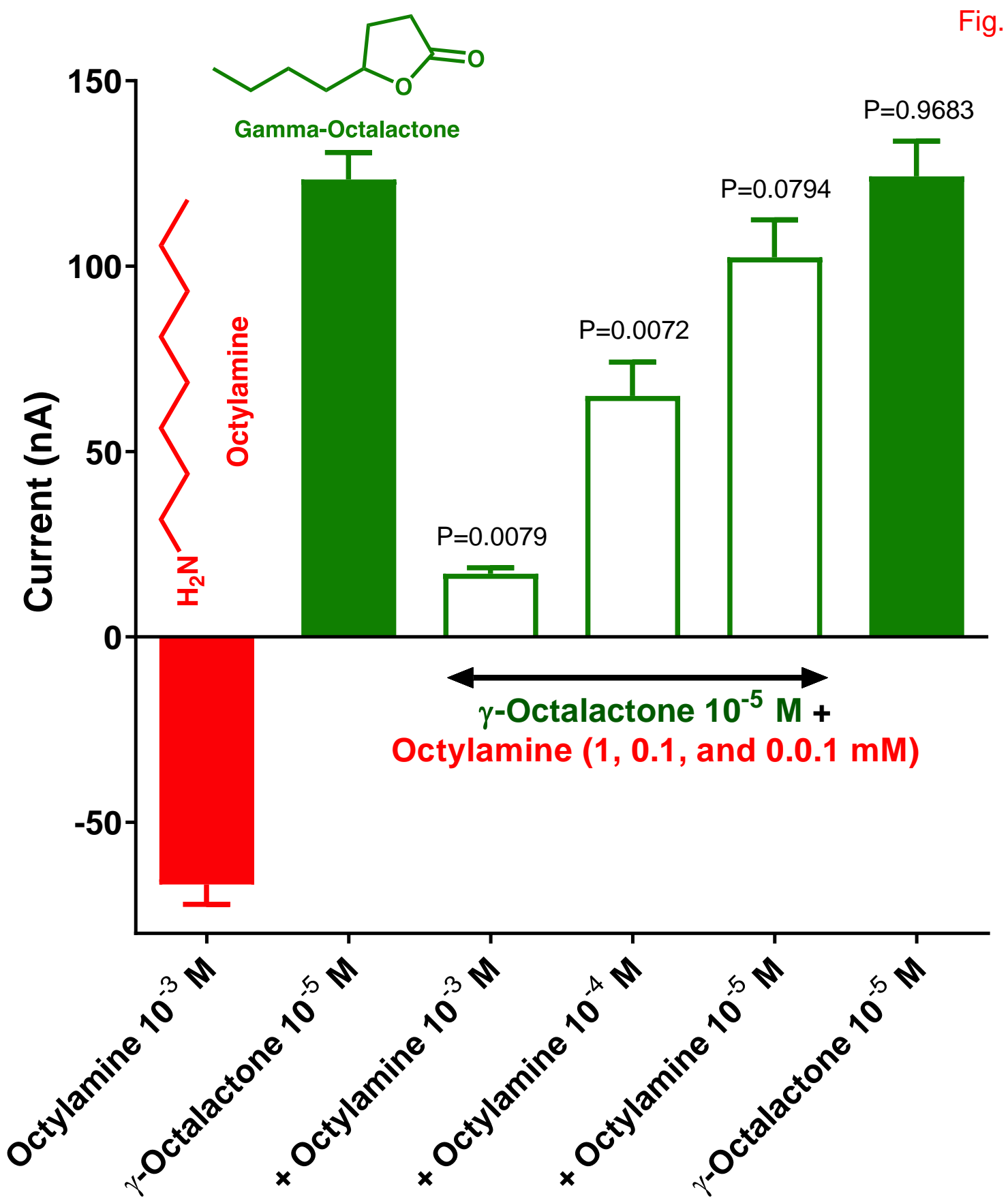



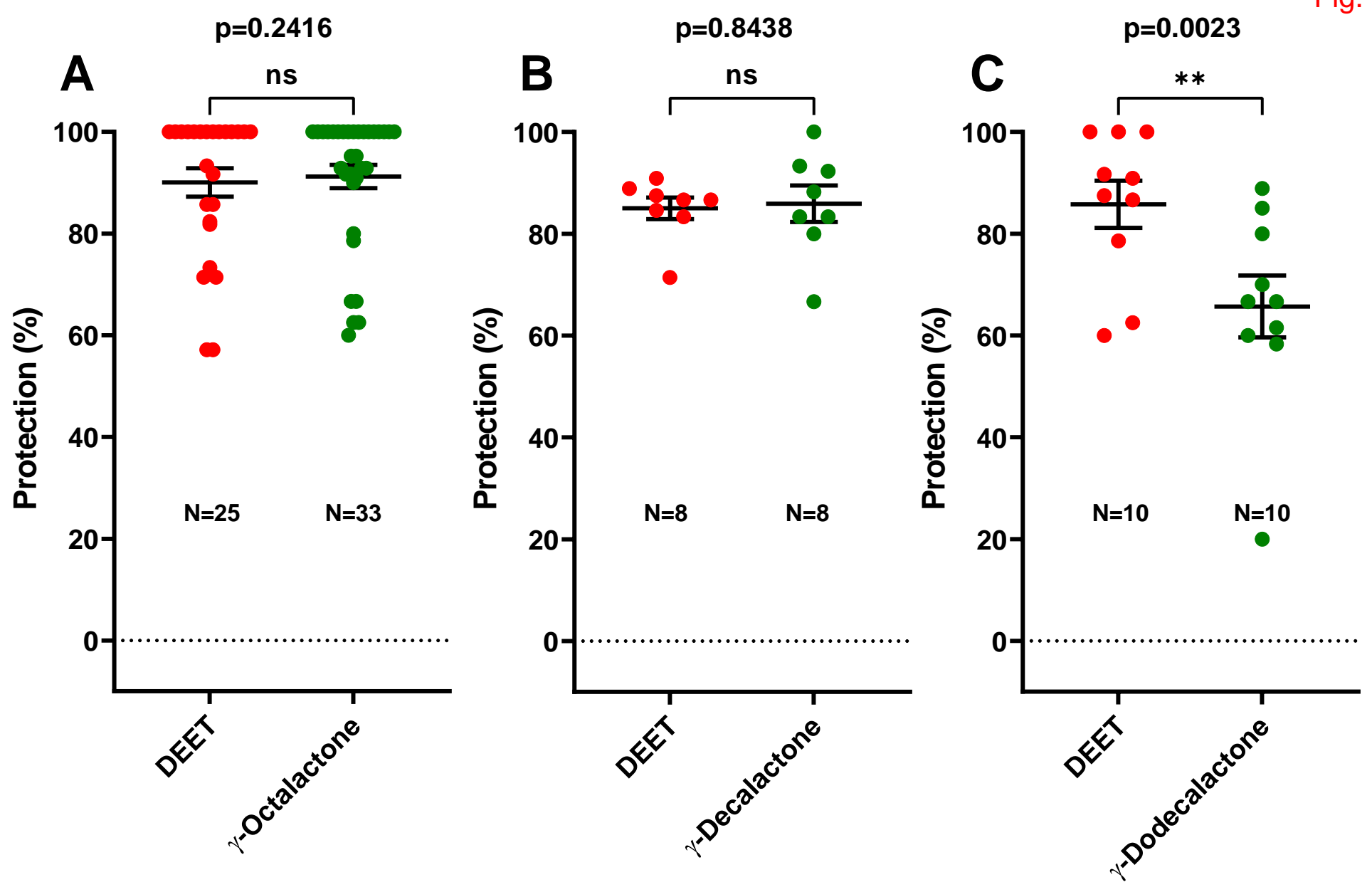


\section{Hand-in-Cage Assay}

Fig. 7

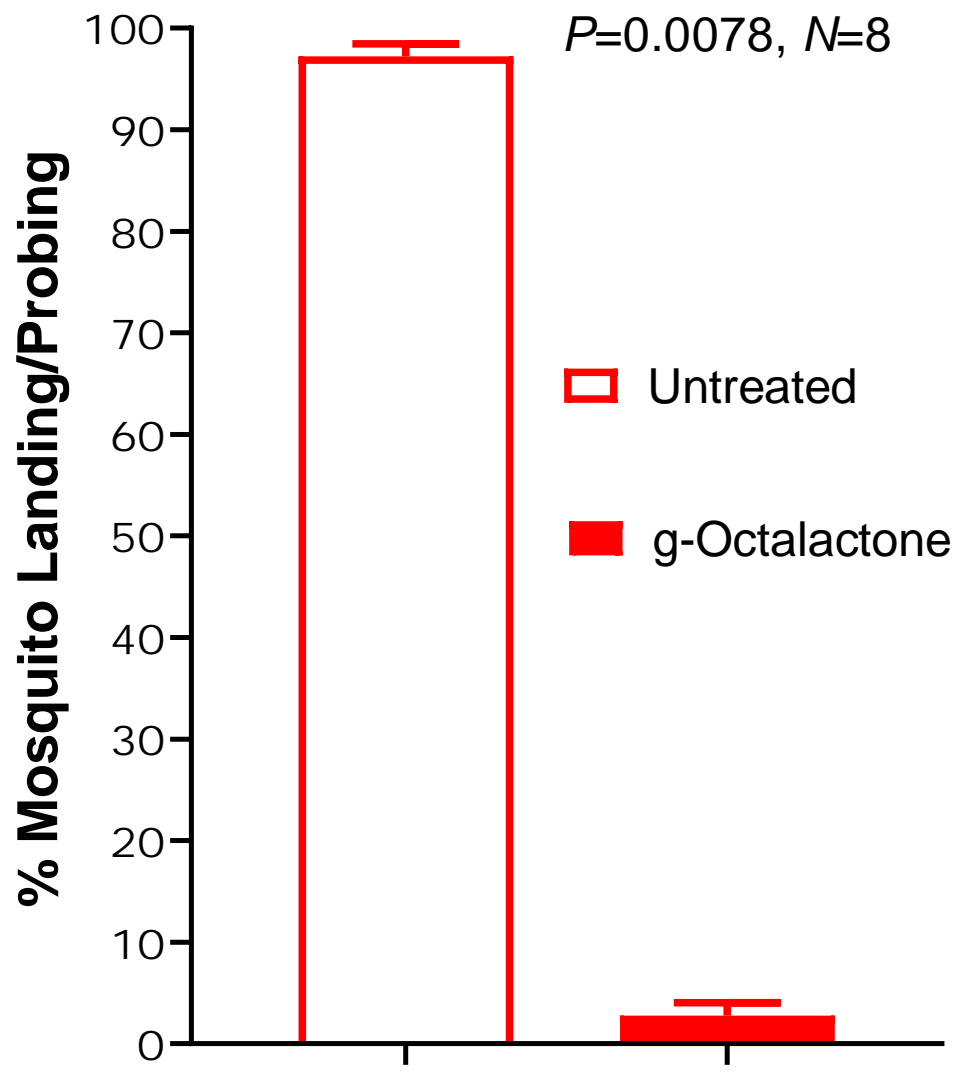

Protection $=97.0 \pm 1.3 \%$ 